\title{
Increased morbidity of obese mice infected with mouse-adapted SARS-CoV-2
}

\author{
Ya-Nan Zhang 1,2, Zhe-Rui Zhang ${ }^{1,2}$, Hong-Qing Zhang ${ }^{1,2}$, Xiao-Dan Li ${ }^{3}$, Jia-Qi Li ${ }^{1,2}$, Qiu-Yan Zhang ${ }^{1}$, Jing Liu ${ }^{1,2}$, Qi Li ${ }^{4}$,

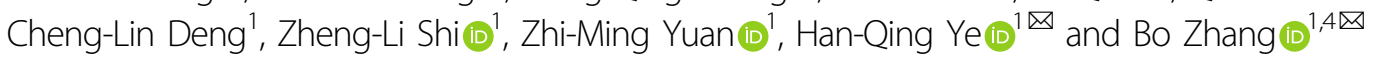

Dear Editor,

Since the outbreak in later 2019, the cofirmed COVID19 cases have surpassed 162 million, with more than 3 million deaths around the world (https://covid19.who.int), inflicting huge impacts on human health and unprecedented shocks to the global economy. The symptoms of COVID-19 can range from asymptomatic/mild to severe depending on the medical conditions of patients. Numerous studies have suggested that obesity and multiple obesity-associated comorbidities are high-risk factors for severe COVID-19,2. For instance, a meta-analysis about their linkage indicated that people with obesity who contracted SARS-CoV-2 were $74 \%$ more likely to be admitted to an ICU, and $48 \%$ more likely to die than people of healthy weight ${ }^{3}$. At the same time, based on data from WHO, globally, about $13 \%$ of adults aged $\geq 18$ years had obese in 2016, and the rate is still rising. Confronting the double threat of a viral and obesity pandemic, it is thus important to understand how obesity heightens the risk of severe COVID-19 symptoms. To develop a mouse model that is able to mimic obesity-associated COVID-19 diseases is an essential prerequisite for the mechanism investigation as well as the therapeutic evaluation of COVID-19 vaccines and antivirals for the obese population.

COVID-19 modeling in mice has been limited by the species differences in entry receptor of SARS-CoV-2, angiotensin converting enzyme 2 protein, between mice and humans ${ }^{4}$. Using mouse-adapted SARS-CoV-2 is one

\footnotetext{
Correspondence: Han-Qing Ye (yehq@wh.iov.cn) or Bo Zhang (zhangbo@wh. iov.cn)

'Key Laboratory of Special Pathogens and Biosafety, Center for Emerging Infectious Diseases, Wuhan Institute of Virology, Chinese Academy of Sciences, Wuhan, China

${ }^{2}$ University of Chinese Academy of Sciences, Beijing, China

Full list of author information is available at the end of the article These authors contributed equally: Ya-Nan Zhang, Zhe-Rui Zhang, Hong-Qing Zhang
}

of efficient solutions to this challenge. In this study, we first obtained a mouse-adapted SARS-CoV-2 (named MP7) through passaging the clinically isolated SARSCoV-2 strain (WIV04) ${ }^{3}$ in the lungs of old BALB/c mice (9-month-old) for 7 rounds (Supplementary Fig. S1a-c). MP7 produced growth curves similar to those of the parental WIV04 virus in Vero-E6 cells at a multiplicity of infection of 0.01 (Supplementary Fig. S1d), albeit with relatively smaller plaques (Supplementary Fig. S1e). In contrast to poor replication of WIV04, MP7 replicated productively in BALB/c mice upon intranasal inoculation with equal amount of viruses $\left(10^{5} \mathrm{PFU}\right)$ characterized by much higher copy numbers of viral RNAs in tissues expecially in lungs, nasal turbinates, and trachea (Supplementary Fig. S2b). Moreover, infection of $10^{3}-10^{5} \mathrm{PFU}$ of MP7 caused $80 \%-100 \%$ mortality rate in old BALB/c mice within the entire experimental period (Supplementary Fig. S2a). Although MP7 was not lethal for young BALB/c mice (8-week-old) (Supplementary Fig. S2c), apparent weight loss (Supplementary Fig. S2d) and efficient viral replication in the lung of infected mice were observed. Complete genome sequencing of MP7 revealed seven specific nucleotide changes that resulted in six nonsynonymous mutations, including two residue substitutions (Q498H and $\mathrm{H} 655 \mathrm{Y})$ in viral spike (S) protein (Supplementary Table S1) that are the mutations emerging frequently in mouse-adapted strains ${ }^{5}$ or during natural SARS-CoV-2 evolution in humans ${ }^{6}$. It is unclear regarding which mutation(s) may contribute to the lethal disease in old BALB/c mice, as there is no common in mutation profiles between the current MP7 strain and the previsouly reported $\mathrm{MA}^{7} \mathrm{7}^{7}$, to our knowledge, that is the only one lethal mouse-adapted strain of SARS-CoV-2 so far.

Then, the association between obesity and the outcomes of COVID-19 was investigated in MP7-based mouse model. 


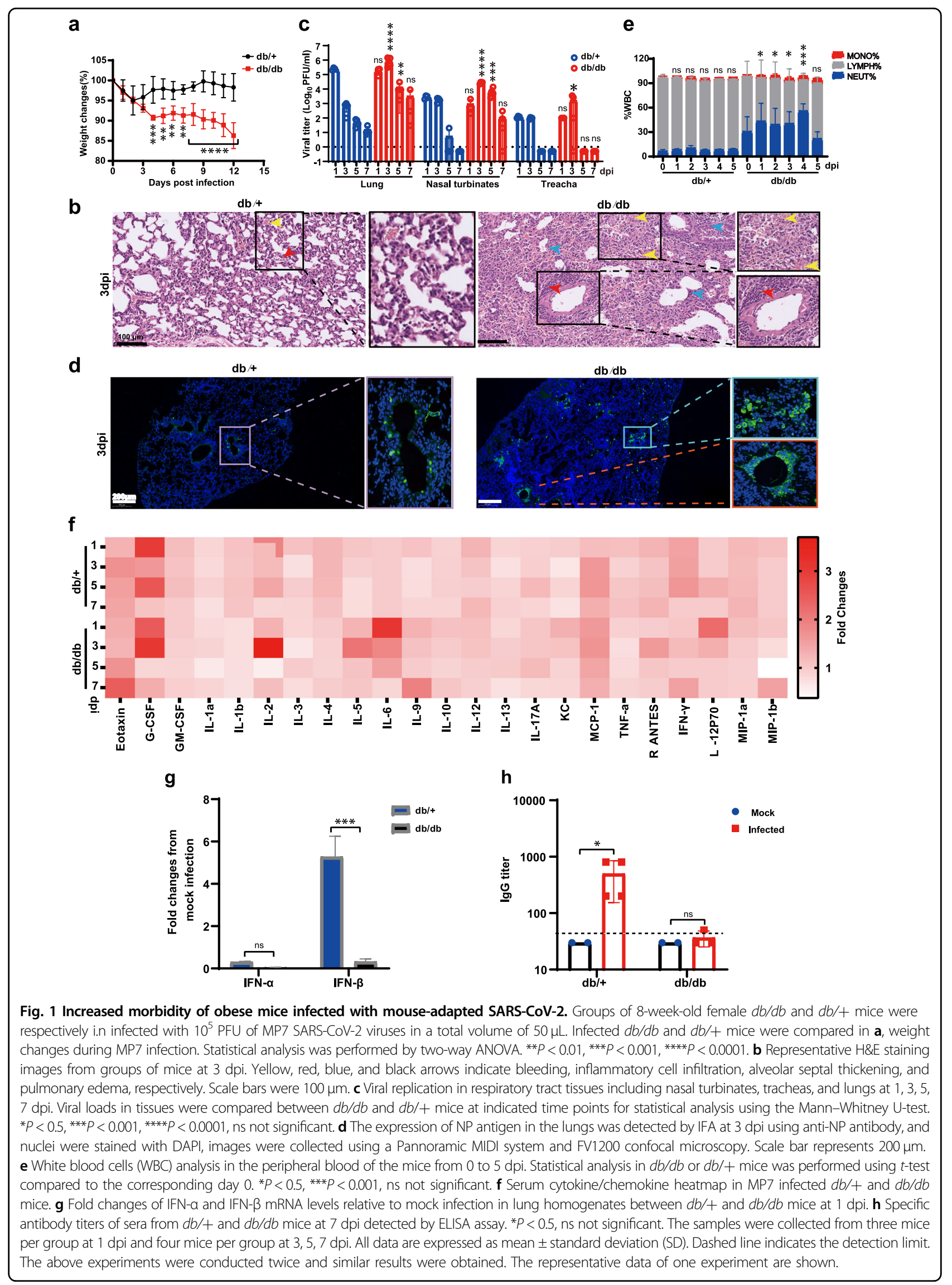


Eight-week-old female C57BL/Ks)- $d b / d b$ mice (a wellestalished mouse model of obesity caused by dysfunctional leptin receptors) and the control C57BL/KsJ- $d b /+$ mice were intranasally inoculated with $10^{5} \mathrm{PFU}$ of MP7. Mice from both groups all survived MP7 infection, but the $d b / d b$ mice developed more severe symptoms than the $d b /+$ mice, including (i) obvious ruffled fur from 2 to 4 days post infection (dpi), (ii) up to $10 \%$ loss of body weight at $4 \mathrm{dpi}$ followed by consisitently low levels of weight till $12 \mathrm{dpi}$ (Fig. 1a), and (iii) massive pulmonary hemorrhage with more macrophage proliferation (yellow arrow), large area of alveolar septal thickening (blue arrow) and infiltration of acute and chronic inflammatory cells near the blood vessels (red arrow) observed on lung sections at 3 dpi by histopathological analysis (Fig. 1b). In contrast, the $d b /+$ mice only experienced about $5 \%$ reduction in body weight during the first $2 \mathrm{dpi}$, and thereafter recovered gradually to the original level (Fig. 1a). In addition, much less lung lesions were observed in $d b /+$ mice at either experimental time point (Fig. 1b and Supplementary Fig. S3a).

At the same time, the viral loads in lungs, nasal turbinates, and trachea were compared between $d b / d b$ and $d b /+$ mice at 1, 3, 5, and 7 dpi through plaque assays (Fig. 1c). MP7 could propagate effeciently in both groups of mice, and about 1-3 log higher levels of viral titers, with some exceptions at early or late time points, were detected in the lungs, nasal turbinates, and trachea of $d b / d b$ mice than those of $d b /+$ mice. Consistantly, higher levels of viral antigens were observed in the lungs of $d b / d b$ mice compared with $d b /+$ mice through immunofluorescence staining with a polyclonal antibody against viral NP protein (Fig. 1d). Notably, in contrast to a sharp decline following peak viral load in $d b /+$ mice, $d b / d b$ mice maintained viral load at a relatively high level for an extended period of time. Such differences of either viral load or its diminished rate between control and obese mice may account for different outcomes of COVID-19 oberved in them.

To further assess clinical obesity-associated severe COVID-19 outcomes on mouse model, the neutrophilslymphocyte ratios (NLRs) in peripheral blood of both groups of mice were measured longitudinally every day from 0 to 5 dpi. Compared with $d b /+$ mice, $d b / d b$ mice not only had a relatively higher baseline NLR due to obesity-caused chronic inflammation ${ }^{8}$, but also yielded more enhancement of NLR upon infection before declining and recovering to baseline level at $5 \mathrm{dpi}$ (Fig. 1e), which is consistent with the manifestations of severe patients. In addition, immunohistochemical staining results also showed that more clustering of neutrophils infiltrated into the lungs of $d b / d b$ mice rather than the $d b /+$ mice at $3 \mathrm{dpi}$ while large numbers of macrophages infiltrated into the lungs of the both mice (Supplementary Fig. S3b). Meanwhile, 23 cytokines/chemokines in sera were quantified using a Luminex cytokine analysis at different time points.
It showed that SARS-CoV-2 infection induced temporal changes in cytokines/chemokines profiles, for instance, Eotaxin, G-CSF, IL-2, IL-5, IL-6, IFN- $\gamma$, IL-9, and MCP-1 expressions were increased in $d b /+$ and $d b / d b$ mice (Fig. 1f). Notably, IL-6, a major inflammatory indicator implicated with the severity of COVID-19,10, was highly elevated in $d b / d b$ mice upon infection followed by gradual decline, but always much higher than that of $d b /+$ mice (except for at the late of infection, $7 \mathrm{dpi}$ ). This result also lends support to IL-6 blockade as a feasible therapeutic agent to treat obese patients infected with SARS-CoV-2. We then measured IFN- $\alpha$ and IFN- $\beta$ mRNA levels in the lungs of infected mice at 1 dpi by qRT-PCR assay (Fig. 1g). It showed that upon infection, (i) IFN- $\alpha$ was not increased in both $d b / d b$ and $d b /+$ mice and there was no significant differences between them; (ii) unlike $d b /+$ mice whose IFN- $\beta$ mRNA was upregulated, the expression IFN- $\beta$ mRNA in $d b / d b$ mice was inhibited (about 0.33-fold relative to mock infection), supporting that severe and critical patients always correlated with impaired type I interferon response'.

Moreover, we compared antibody responses to SARS$\mathrm{CoV}-2 \mathrm{~S}$ protein between both groups (Fig. 1h) using ELISA. A much poorer antibody response was observed in $d b / d b$ mice despite higher viral replication in the respiratory tract, arguing the necessity to weigh COVID19 vaccine efficacies in obese people.

Overall, our study provides a mouse model demonstrating obesity-associated COVID-19 comorbidities using a mouse-adapted SARS-CoV-2 strain, MP7. It will help elucidate mechanisms of pathogenesis that may be occurring in humans with obesity and accelerate the development of therapeutics for this highly susceptible population, although a longer-term outcome evaluation may be expected to provide more comprehensive information.

\section{Acknowledgements \\ The experiments related to SARS-CoV-2 were completed at National Biosafety Laboratory, Wuhan, Chinese Academy of Sciences. We are particularly grateful to the staffs from Zhengdian BSL-3 Laboratory (Tao Du, Jin Xiong and Lun Wang), Center for Animal Experiment (Yan-Feng Yao, Ge Gao,Yun Peng and Jing Deng), and the running team of the laboratory for their helpful supports during the course of the work. This work was supported by the National Key Research and Development Program of China (2018YFA0507201).}

\section{Author details \\ ${ }^{1}$ Key Laboratory of Special Pathogens and Biosafety, Center for Emerging Infectious Diseases, Wuhan Institute of Virology, Chinese Academy of Sciences, Wuhan, China. ${ }^{2}$ University of Chinese Academy of Sciences, Beijing, China. ${ }^{3}$ School of Medicine, Hunan Normal University, Changsha, China. ${ }^{4}$ Drug Discovery Center for Infectious Diseases, Nankai University, Tianjin, China}

\section{Author contributions}

B.Z. and H.-Q.Y. designed and coordinated the study, analyzed and interpreted the results; Y.-N.Z., Z.-R.Z., and H.-Q.Z. performed most parts of experiments with the help from X.-D.L., J.-Q.L., Q.-Y.Z., J.L., Q.L. and C.-L.D.; Z.-L.S. and Z.-M.Y. provided reagents for experiments. 


\section{Data and materials availability}

All data are available in the main text or the supplementary materials.

\section{Conflict of interest}

The authors declare no competing interests.

\section{Publisher's note}

Springer Nature remains neutral with regard to jurisdictional claims in published maps and institutional affiliations.

Supplementary information The online version contains supplementary material available at https://doi.org/10.1038/s41421-021-00305-x.

Received: 11 February 2021 Accepted: 11 July 2021

Published online: 25 August 2021

\section{References}

1. Drucker, D. J. Diabetes, obesity, metabolism, and SARS-CoV-2 infection: the end of the beginning. Cell Metab. 33, 479-498 (2021).
2. Drucker, D. J. Coronavirus infections and Type 2 diabetes-shared pathways with therapeutic implications. Endocr. Rev. 41, bnaa011 (2020).

3. Popkin, B. M. et al. Individuals with obesity and COVID-19: a global perspective on the epidemiology and biological relationships. Obes. Rev. 21, e13128 (2020).

4. Zhou, P. et al. A pneumonia outbreak associated with a new coronavirus of probable bat origin. Nature 579, 270-273 (2020).

5. Wang, J. et al. Mouse-adapted SARS-CoV-2 replicates efficiently in the upper and lower respiratory tract of BALB/C and C57BL/6J mice. Protein Cell 11, 776-782 (2020).

6. Laha, S. et al. Characterizations of SARS-CoV-2 mutational profile, spike protein stability and viral transmission. Infect. Genet. Evol. 85, 104445 (2020).

7. Leist, S. R. et al. A mouse-adapted SARS-CoV-2 induces acute lung injury and mortality in standard laboratory mice. Cell 183, 1070-1085 (2020).

8. Aydin, M. et al. Neutrophil/lymphocyte ratio in obese adolescents. North Clin. Istanb. 2, 87-91 (2015).

9. Hadjadj, J. et al. Impaired type I interferon activity and inflammatory responses in severe COVID-19 patients. Science 369, 718-724 (2020).

10. Zhu, Z. et al. Clinical value of immune-inflammatory parameters to assess the severity of coronavirus disease 2019. Int J. Infect. Dis. 95, 332-339 (2020) 